\title{
Evidence for a human-specific Escherichia coli clone
}

\author{
Olivier Clermont, ${ }^{1}$ Mathilde Lescat, ${ }^{1}$ \\ Claire L. O'Brien, ${ }^{2}$ David M. Gordon, ${ }^{2}$ \\ Olivier Tenaillon ${ }^{1}$ and Erick Denamur ${ }^{1 *}$ \\ ${ }^{1}$ INSERM U722 and Université Paris 7 Denis Diderot, \\ France. \\ ${ }^{2}$ School of Botany and Zoology, The Australian National \\ University, Canberra, ACT 0200, Australia.
}

\section{Summary}

Escherichia coli is a widespread commensal of the vertebrate intestinal tract. Until recently, no strong association between a particular clone and a given host species has been found. However, members of the B2 subgroup VIII clone with an 081 serotype appear to be human host specific. To determine the degree of host specificity exhibited by this clone, a PCR-based assay was used to screen 723 faecal and clinical isolates from humans, and 904 faecal isolates from animals. This clone was not detected among the animal isolates, but was discovered in people living in Africa, Europe and South America. The clone is rarely isolated from people suffering from intestinal or extraintestinal disease and is avirulent in a mouse model of extraintestinal infection. Fine-scale epidemiological analysis suggests that this clone is competitively dominant relative to other members of the B2 phylogenetic group and that it has increased in frequency over the past $\mathbf{2 0}$ years. This clone appears to be a good candidate for use as a probiotic, and may be suitable as an indicator of human faecal contamination in microbial source tracking studies.

\section{Introduction}

Escherichia coli is a widespread commensal of the lower intestinal tract of humans and other vertebrates that occasionally causes intestinal and extraintestinal diseases (Donnenberg, 2002). Understanding what makes E. coli an occasionally devastating pathogen requires a better knowledge of its ecology as a commensal. A first step in gaining this knowledge would be to determine if niche specialization occurs within the species. E. coli colonizes a wide variety of hosts with very diverse gut morphologies and digestive physiologies. E. coli also exhibits substan-

Received 01 August, 2007; accepted 31 October, 2007. ${ }^{*}$ For correspondence. E-mail denamur@ bichat.inserm.fr; Tel. (+33) 144856156 ; Fax (+33) 144856149. tial genetic structure, with most faecal isolates belonging to one of four genetic groups (A, B1, D and B2) (Herzer et al., 1990; Wirth et al., 2006). However, epidemiological studies have only demonstrated weak associations between host species and the prevalence of strains of the four phylogenetic groups (Gordon and Cowling, 2003; Escobar-Páramo et al., 2006). Although the frequency of the four genetic groups varies to some degree with the diet or body mass of the host from which they are isolated, other factors such as climate, year of sampling, or the domestication status of the animals sampled (wild versus domesticated) also shape the genetic structure of $E$. coli. To date, no strong association between a particular clone and a given host species has been found, except perhaps for a hly B1 clone that appears to be restricted to animals (Escobar-Páramo et al., 2006).

The great majority of $E$. colistrains belonging to genetic group B2 are highly virulent in a mouse model of extraintestinal infection (Johnson et al., 2006). Recently, as part of a detailed determination of the genetic makeup of group B2 strains, we identified an unusual B2 strain, in that it is avirulent in the mouse model of extraintestinal infection (Le Gall et al., 2007). This strain has an 081 serotype and is a member of the B2 clonal subgroup VIII as determined by multilocus sequence typing (MLST) (Le Gall et al., 2007). Preliminary epidemiological evidence suggested that this strain might be human specific. Consequently, we undertook a large-scale epidemiological survey with the goal of determining the distribution of this strain in human and non-human vertebrates across four continents.

\section{Results}

\section{Global distribution of the B2 VIII/O81 clone}

A total of 1369 strains belonging to phylogenetic group $\mathrm{B} 2$, representing 438 faecal and 285 clinical isolates from humans, and 646 faecal isolates from wild, domesticated and zoo animals, were screened using the 081-specific primer. An additional 258 faecal isolates from nondomesticated animals and 15081 strains from septicemic chickens, all of unknown phylogenetic group, were also screened. All 081-positive strains were screened using the B2 clonal subgroup VIII-specific primers and, if unknown, their phylogenetic group membership was determined.

Despite surveying strains from over 60 animal species living on four continents, none of the animal isolates were 
found to possess the $\mathrm{O} 81$ locus and to be members of B2 clonal subgroup VIII. By contrast, 64 members of the B2 $\mathrm{VIII/O81} \mathrm{clone} \mathrm{were} \mathrm{detected} \mathrm{among} \mathrm{the} \mathrm{human} \mathrm{isolates.}$ Two of the B2 VIII/O81 strains were clinical isolates; one strain (IAI48) was responsible for a urinary tract infection (Picard et al., 1999) and the other (381A) was an enteroaggregative strain responsible for a case of diarrhoea (Escobar-Páramo et al., 2004a). B2 VIII/O81 strains were isolated from humans living in Africa, the Americas and Europe, but not from people living in Australia.

Seven B2 strains from both non-human vertebrates and humans yielded an 081 polymerase chain reaction (PCR) product but were not members of the B2 clonal subgroup VIII, indicating that the 081 serotype can be found in other B2 clonal groups. Further, three O81-positive strains belonging to phylogenetic groups $A$ and $B 1$ were detected from mammals living in the Americas (1 group $A$ and 2 group B1 strains), as well as 15081 B1 strains from chickens sampled in Spain. All of the 081 strains that were not members of the B2 VIII/O81 clone exhibited a polymorphic pattern of virulence determinants, that was, in every case, distinct from the B2 VIII/O81 clone (data not shown).

To assess degree of sequence type diversity among the B2 VIII/O81 strains, we choose six isolates from different hosts and geographical regions, as well as the two disease causing isolates (Table 1) for further characterization. We first performed MLST by sequencing six essential genes (5921 bp) in these strains. The sequences were all identical except for one strain (Ben4d) that has a synonymous polymorphism in the putP gene.

We also performed an in silico search for strains of the B2 VIII/O81 clone in the Max Planck Institute MLST database (http://web.mpiib-berlin.mpg.de/mlst/dbs/Ecoli). This database contained, at the time it was searched, data for 1404 disease and faecal isolates largely recovered from humans and domesticated animals. For this search, the allele sequence for each of the seven genes used in this MLST scheme (adk, fumC, gyrB, icd, mdh, recA and purA) was determined for the B2 VIII/O81 strain ED1a. Only one strain (M716, ST 452), an isolate from an Australian mammal, matched the profile of ED1a. However, this isolate does not yield an O81 PCR product, and M716 differs from ED1a at seven bases over the 727 nucleotides of $\operatorname{trp} A$ locus, including the allele-specific site of the trpAVIII.f primer, as well as in its virulence profile.

\section{Virulence characteristics of B2 VIII/O81 strains}

The eight B2 VIII/O81 strains were tested in an experimental mouse model of extraintestinal virulence. Whereas most B2 strains exhibit a high virulence in such a model (Johnson et al., 2006), none of the tested strains

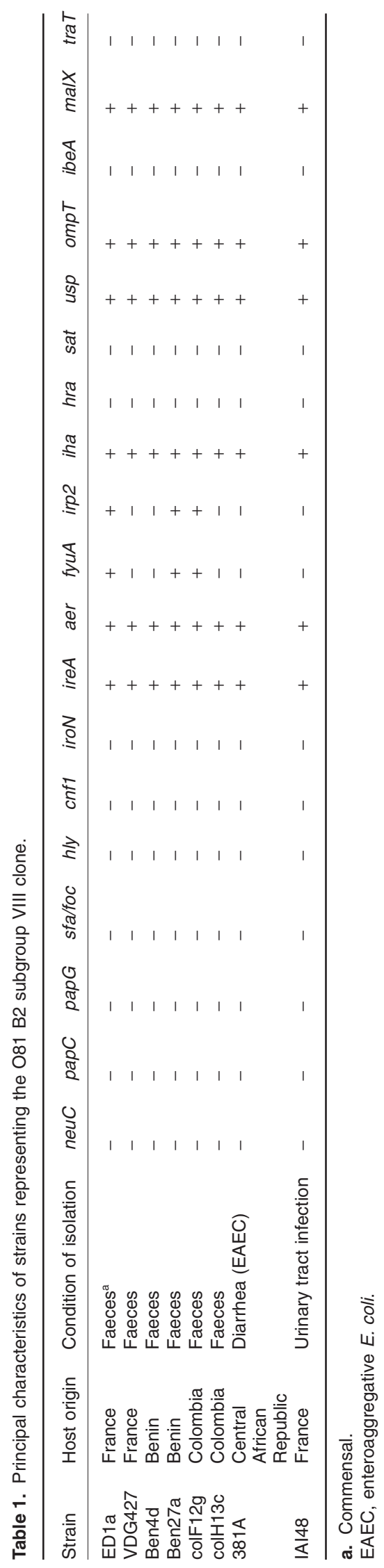

(C) 2008 The Authors

Journal compilation (C) 2008 Society for Applied Microbiology and Blackwell Publishing Ltd, Environmental Microbiology, 10, $1000-1006$ 
had any virulence. We then screened the isolates for the presence of 19 putative virulence determinants associated with extraintestinal disease. All the strains exhibited a similar virulence gene profile (Table 1). The iron metabolism genes ire $A$, aer and iha were present in all strains. However, the genes fyuA and irp2, which are markers for the high-pathogenicity island, were absent from some strains. None of the genes classically associated with intraintestinal virulence was present in the ED1a reference strain.

\section{Fine-scale analysis of the epidemiologic data}

In a sample of faecal isolates collected post 2000 from 152 people living in Colombia, Benin, and France, and considering one randomly selected isolate per individual, the B2 VIII/O81 clone represented $4 \%$ of all isolates (3-7\% according to the population) and $18 \%$ of the B2 strains recovered (11-28\% according to the population). By contrast, in a sample of faecal isolates collected in the 1980s from 193 people living in the USA, Mali, Croatia, France and Sweden, the B2 VIII/O81 clone represented only $0.4 \%$ of all isolates tested and $4 \%$ of the B2 strains. This result suggests that the VIII/O81 clone has increased its representation in human faeces 10 -fold in the past 20 years.

To determine if the B2 VIII/O81 clone exists at a high frequency within a host we analysed the data set cited above, but used all 10 isolates taken from each of the 152 subjects. 081 was detected in 12 of the 152 hosts and, in $42 \%$ of these people, the 081 clone represented at least $80 \%$ of the $E$. coli isolates recovered from that host. By contrast a non-VIII/O81 B2 strain was detected in 58 hosts, but in these cases the B2 strain represented $>80 \%$ of the E. coli in that host only $17 \%$ of the time. Put differently, $77 \%$ of the 081 strains detected were found in hosts in which they were the majority clone $(>80 \%)$, whereas only $37 \%$ of non-O81 B2 strains were found in a situation where they represented the majority $(>80 \%)$ of the $E$. coli in a host. This outcome suggests that the B2 VIII/O81 clone is able to achieve numerical dominance in a human more often than other group B2 strains. Furthermore, in one subject, all 30 isolates taken from faeces emitted at three different times (10 isolates per faeces) over a 1-year period as well as 10 isolates from stools obtained 6 years after the first sample were the B2 VIII/O81 clone. This result demonstrates that this clone can persist in the human gut for extend periods.

\section{Survival in water}

A human-specific strain of $E$. coli could be a useful marker in microbial source-tracking studies that attempt to deter- mine if human faecal contamination of a water body is occurring. Consequently, we tested the survival of ED1a and compared it with the survival of $E$. coli K12 (Bogosian et al., 1996). In non-sterile river water at $20^{\circ} \mathrm{C}$ the decline in viable cell counts of both strains are identical, and by day 9 neither strain can be detected (Fig. 1A). This result is in accord with previously reported outcomes for $\mathrm{K} 12$ (Bogosian et al., 1996). In sterile river water at $20^{\circ} \mathrm{C}$, viable cell counts of both strains declined by only 1-2 log in 35 days, with ED1a exhibiting poorer survival than K12 (Fig. 1B). These findings are consistent with earlier suggestions that the decline of $E$. coli populations in water is due to predation by protozoa, phages and exposure to heat-labile toxins.

\section{Discussion}

Host-specific E. coli pathogens are not unknown, for example it has long been thought that Shigella strains are primate specific (Donnenberg, 2002) and there are rabbitspecific enteropathogenic E. coli (Leyton et al., 2007). However, this is the first report of what is apparently a human host-specific commensal strain of $E$. coli, although it is not known if this strain can colonize other primates. This clone belongs to the B2 phylogenetic group and, although B2 strains are often responsible for extraintestinal infection (Picard et al., 1999), strains of this clone appear to rarely cause disease. Only two pathogenic strains belonging to this clone have been isolated so far, one strain causing a urinary tract infection and an enteroaggregative strain. The B2 VIII/O81 clone is absent from the Acthman E. coli MLST database and over half of the more than 1400 isolates in this database are listed as pathogens (Wirth et al., 2006). Based on its virulence determinant pattern and/or serotype, this clone is also absent from European and American collections of B2 strains responsible for newborn meningitis ( $n=99$, Bonacorsi et al., 2003) and urosepsis $(n=108$, Johnson and Stell, 2000; Bingen-Bidois et al., 2002). Finally, none of the strains of this clone is virulent in the mouse model of extraintestinal infection.

B2 VIII/O81 clone appears to be highly successful as it has been found on three continents and, in some populations, it can represent up to $7 \%$ of the isolates taken from human faeces. The reason for the absence of this clone in humans living in Australia is unknown. Geographic isolation is unlikely to be the answer, as over $60 \%$ of the MLST sequence types isolated from Australian humans have been detected in other parts of the world (D. Gordon unpubl. data). All isolates of the B2 VIII clone with an 081 serotype are also very homogeneous in that they possess a very similar suit of genes, while in other B2 strains these genes have a highly polymorphic distribution (this work and Le Gall et al., 2007). These observations 
A

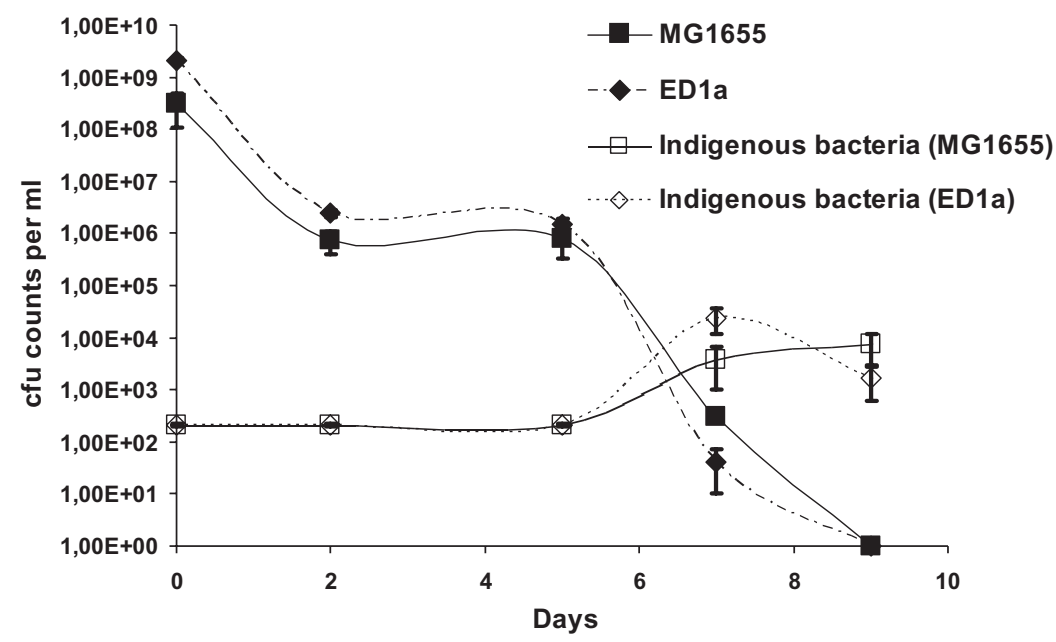

Fig. 1. Decline of K12-MG1655 and ED1a E. coli cells in $(A)$ non-sterile and $(B)$ sterile water of the Saint Martin canal in Paris at $20^{\circ} \mathrm{C}$. Indigenous bacteria counts are indicated in the non-sterile experiment.

B

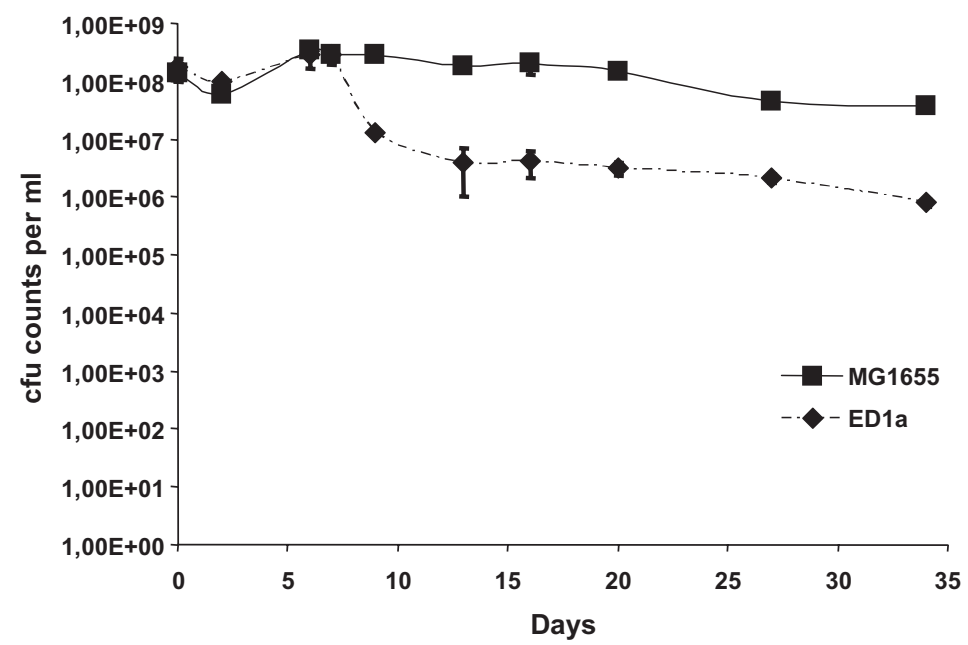

are consistent with the idea that the clone has undergone a recent expansion.

The existence of a human-specific $E$. coli clone has potential applied applications. The B2 VIII/O81 clone could be used as a probiotic, as it appears to be avirulent and good colonizer of the human gut. The avirulent nature of this strain means that it might be particularly appropriate for use in highly vulnerable patients, such as those in intensive care, the immunocomprised, or premature infants. By comparison, the currently used probiotic strains, Nissle 06 strain (Mutaflor $^{\circledR}$ ) isolated in 1917 from the faeces of a healthy soldier (Sun et al., 2005) and the A0 34/86 O83 strain isolated from porcine faeces (Hejnova et al., 2005), are highly virulent in our mouse model of extraintestinal virulence. Nissle 06 killed all 10 inoculated mice and A0 34/86 O83 killed nine of the 10 mice inoculated.
Microbial source tracking could be another application of the O81/B2 subgroup VIII clone. Coliforms and E. coli, in particular, have been used for many years, to assess both water quality and to determine the source of any faecal pollution that might occur in a water body (Scott et al., 2002). However, the use of E. coli in these efforts has been hampered by the apparent lack of any extensive host specificity in E. coli (Gordon, 2001) and by the ability of many $E$. coli strains to undergo significant cell division in the external environment (Barnes and Gordon, 2004; Power et al., 2005). The usefulness of the microbial indicators as a tool in risk assessment can be significantly enhanced by the identification of a human-specific clone. The fact that not all individuals carry the O81/B2 subgroup VIII clone is not restrictive, as human faecal pollution is generally due to release of sewage from wastewater treatment plants or septic tanks, and therefore represents 
faecal contamination not by individuals but by populations. The inability of the B2 subgroupVIII/O81 clone to undergo significant cell division in water makes it a good candidate marker of human faecal contamination of water. Furthermore, the PCR assay that we have developed can be used to identify the clone directly from water or soil samples.

We have identified an E. coli clone that is an excellent candidate for further molecular investigation aimed at understanding (i) what defines a commensal $E$. coli strain and (ii) what attributes of the strain are responsible for its host specificity. Consequently, we have initiated a project whose aim is to sequence the genome of strain ED1a (http://www.genoscope.cns.fr/externe/English/Projets/ Projet_MW/organisme_MW.html) in conjunction with an in-depth metabolic characterization of this strain.

\section{Experimental procedures}

\section{Bacterial strains}

Several previously published collections were studied. A collection of 293 isolates representing all of the B2 strains among 1520 isolates taken from the faeces of 152 humans ( 10 strains per individual) (Escobar-Páramo et al., 2004b). These strains originated from Benin (68 isolates/24 subjects), Colombia (81 isolates/18 subjects) and France (144 isolates/25 subjects) and were obtained post 2000 . Twenty-five human faecal B2 isolates from 25 subjects originating from Europe (France, Sweden, Croatia), Africa (Mali) and America (USA) and sampled in the 1980s (Ochman and Selander, 1984; Duriez et al., 2001) were also studied. A collection of 378 faecal isolates (310 isolates from 87 mammals, 68 isolates from 23 birds) representing all of the B2 strains among 1930 isolates from the faeces of 405 animals (one to 10 strains per individual). These strains were sampled between 1980 and 2000 in France, Africa (Ethiopia, Cameroon, Gabon) and the Americas (French Caribbean Islands, Venezuela, USA) from domesticated and wild (Escobar-Páramo et al., 2006), as well as zoo (Ochman and Selander, 1984) animals. A collection of 55 pathogenic B2 isolates from humans (one strain per patient) encompassing enteropathogenic, enteroaggregative, diffusely adhering and extraintestinal pathovars (Ochman and Selander, 1984; Picard et al., 1999; Escobar-Páramo et al., 2004a). These strains originated from France, Sweden, Brazil, USA and Central African Republic, and were collected between 1980 and 2000. The collection of strains isolated from humans living in the Canberra region of Australia was acquired by isolating a single colony from the faeces or an extraintestinal body site of each person sampled (Gordon et al., 2005; Gordon and O'Brien, 2006). A total of 120 B2 faecal isolates and 230 B2 strains taken from extraintestinal body sites were examined for this study. The collection of strains from native wild Australian vertebrates was acquired by selecting a single isolate from each faecal sample and a single faecal sample was taken from each host individual (Gordon and Cowling, 2003). The numbers of Australian B2 strains examined were: mammals $(n=234)$, birds $(n=30)$ and reptiles $(n=4)$. A collection described by Souza and colleagues (1999) and con- sisting of 258 E. coli faecal isolates from animals was analysed. For the Souza collection a single isolate per host was taken from faecal material of the following host types: mammals $(n=197)$, birds $(n=51)$ and reptiles $(n=10)$ living primarily in Central and South America. Lastly, 15081 E. coli strains isolated from the hearts of 15 chickens suffering from septicaemia in Spain during the 1990s (Blanco et al., 1997) were also studied.

\section{PCR screening of O81and B2 subgroup VIII strains}

The serotype of the B2 clonal subgroup VIII strain ED1a was determined via traditional serology (Dr Chobi Deb Roy, E. coli reference center, Pennsylvania State University, USA). To design an 081-specific primer pair, the approach described by Clermont and colleagues (2007) was used. First, the $5^{\prime}$ extremity of the rfb cluster from ED1a was sequenced and using this sequence data an O81-specific primer was designed (rfbO81bisr: 5'-GAGCAGTATATATTACTGGTG-3'). This primer, together with the gndbis.f primer (5'-ATACC GACGACGCCGATCTG-3'), yields a 383 bp fragment.

Primers to confirm that a strain was a member of the B2 clonal subgroup VIII were designed for the trpA gene. The forward primer (trpAVIII.f: 5'-GCGCAAAGAAGGCGCATTCA$3^{\prime}$ ) is specific to B2 subgroup VIII strains and together with a non-specific trpA reverse primer (trpA2.r: 5'-GCAACGCGG CCTGGCGGAAG-3') produces a 394 bp fragment. The universal forward primer targeting the $3^{\prime}$ portion of $\operatorname{trpB}$ (trpBA.f: 5'-CGGCGATAAAGACATCTTCA-3') which, together with the trpA2.r primer, yields a 489 bp product from all $E$. coli strains was included in the reaction as a positive control.

Polymerase chain reactions were carried out in a $20 \mu \mathrm{l}$ volume and contained $2 \mu \mathrm{l}$ of $10 \times$ buffer (supplied with Taq polymerase), $20 \mathrm{pmol}$ of each primer, $2 \mu \mathrm{M}$ each dNTP, $1 \mathrm{U}$ of Taq polymerase (Ozyme, St Quentin en Yvelines, France), and $3 \mu \mathrm{l}$ of bacterial lysate. Polymerase chain reactions were performed with an Eppendorf Mastercycler with MicroAm tubes using the following amplification conditions: $4 \mathrm{~min}$ at $94^{\circ} \mathrm{C}, 30$ cycles of $5 \mathrm{~s}$ at $94^{\circ} \mathrm{C}$ and $10 \mathrm{~s}$ at $59^{\circ} \mathrm{C}$, with a final extension step of $5 \mathrm{~min}$ at $72^{\circ} \mathrm{C}$. Polymerase chain reaction products were loaded on $2 \%$ agarose gels containing SYBR ${ }^{\circledR}$ Safe DNA gel stain (Invitrogen, Cergy Pontoise, France) and visualized and photographed under UV light.

\section{MLST analysis}

The phylogenetic relationships among the strains were inferred using nucleotide sequence data from six essential genes [trpA, $\operatorname{trp} B, p a b B$, putP, icd, and polB (Gerdes et al., 2003)] which are thought to experience little recombination and produce a strong phylogenetic signal (Escobar-Páramo et al., 2004c).

\section{Virulence gene detection}

The following 19 genes, usually considered as extraintestinal 'virulence determinants' and involved in capsule synthesis (neuC), adhesion (papC, papG, sfa/foc), toxin production (hly, cnf1), iron metabolism (iron, ireA, aer, aer, fyuA, irp2, 
iha) and other traits (hra, sat, usp, ompT, ibeA, malX, traT), were detected by using established simplex or multiplex PCR assays (Johnson et al., 2006). An in silico search of the complete genome of B2 subgroup VIII strain ED1a (V. Barbe, C. Médigue, E. Denamur unpubl. data) was undertaken to determine if this strain harboured genes also found in the main pathovars of diarrhoeagenic E. coli, i.e. diffusely adhering E. coli ( $a f a B C / d a a C)$, enteroaggregative E. coli (AA probe), enterohemorragic E. coli and enteropathogenic E. coli (eae), enteropathogenic E. coli ( $b f p A)$, enterotoxigenic E. coli (STa and LT-1), enterohemorragic E. coli (stx1, stx2, and $e h x A$ ) and enteroinvasive E. coli (ipaB) (Escobar-Páramo et al., 2004a).

\section{Survival of E. coli strains in water}

The river water used was obtained from the Saint Martin canal in Paris at a site about $1 \mathrm{~km}$ upstream from the Seine. For the sterile water studies, the water was autoclaved for $45 \mathrm{~min}$. About $100 \mathrm{ml}$ aliquots of water were placed into $250 \mathrm{ml}$ Erlenmeyer flasks capped with paper covers. After inoculation, the flasks were incubated at $20^{\circ} \mathrm{C}$ under a $12: 12$ light : dark cycle. Indigenous bacteria were present at a level of about $2 \times 10^{2} \mathrm{cfu} \mathrm{ml}^{-1}$ of river water. Fresh cultures of the strains K12-MG1655 and ED1a that have been grown for $14 \mathrm{~h}$ in $10 \mathrm{ml}$ of Luria-Bertani (LB) medium were washed with sterile $0.9 \%$ saline, centrifuged, re-suspended in $1 \mathrm{ml}$ and added to the water. Samples for counting were removed directly from the water, diluted and plated on Drigalsky and LB (non-sterile water) or LB (sterile water) media. In the non-sterile water experiment, the differentiation between indigenous and inoculated bacteria was based on colony morphology and O81 PCR assay. All experiments were carried out in duplicate.

\section{Mouse lethality assay}

A mouse model of systemic infection was used to assess the intrinsic virulence of the 081 strains (Picard et al., 1999). For each strain, 10 outbred female Swiss OF1 mice (3-4 weeks old, 14-16 g) were challenged subcutaneously in the abdomen with a standardized bacterial inoculum $\left(10^{9} \mathrm{cfu} \mathrm{ml}^{-1}\right.$ of log-phase bacteria in $0.2 \mathrm{ml}$ Ringer solution). Mortality was assessed over 7 days post challenge. The urosepsis strains CFT073 was used as a positive control and the faecalderived strain K12-MG1655 as a negative control. Data were available for three strains (ED1a, Ben4d, IAI48) (Le Gall et al., 2007). In this model system, lethality is a rather clearcut parameter and strains were usually classified as non-killer (strains killing $<2$ mice out of 10) or killer (strains killing $>8$ mice) (Johnson et al., 2006).

\section{Acknowledgements}

We are grateful to Maryvonne Moulin-Schouleur and Jorge Blanco for providing the chicken 081 strains and to Valeria Souza for some animal strains. This work was partially funded by the 'Fondation pour la Recherche Médicale'.

\section{References}

Barnes, B., and Gordon, D. (2004) Coliform dynamics and the implications for source tracking. Environ Microbiol 6: 501-509.
Bingen-Bidois, M., Clermont, O., Bonacorsi, S., Terki, M., Brahimi, N., Loukil, C., et al. (2002) Phylogenetic analysis and prevalence of urosepsis strains of Escherichia coli bearing pathogenicity island-like domains. Infect Immun 70: 3216-3236.

Blanco, J.E., Blanco, M., Mora, A., and Blanco, J. (1997) Production of toxins (enterotoxins, verotoxins, and necrotoxins) and colicins by Escherichia coli strains isolated from septicemic and healthy chickens: relationship with in vivo pathogenicity. J Clin Microbiol 35: 2953-2957.

Bogosian, G., Sammons, L.E., Morris, P.J.L., O’Neil, J.P., Heitkamp, M.A., and Weber, D.B. (1996) Death of the Escherichia coli K-12 strain W3110 in soil and water. Appl Environ Microbiol 62: 4114-4120.

Bonacorsi, S., Clermont, O., Houdouin, V., Corevant, C., Brahimi, N., Marecat, A., et al. (2003) Molecular analysis and experimental virulence of French and North American Escherichia coli neonatal meningitis isolates: identification of a new virulent clone. J Infect Dis 187: 1895-1906.

Clermont, O., Johnson, J.R., Menard, M., and Denamur, E. (2007) Determination of Escherichia coli O types by allelespecific polymerase chain reaction: application to the $O$ types involved in human septicemia. Diagn Microbiol Infect Dis 57: 129-156.

Donnenberg, M. (2002) Escherichia coli virulence mechanisms of versatile pathogen. Elsevier Science Ed ed, San Diego, CA, USA.

Duriez, P., Clermont, O., Bonacorsi, S., Bingen, E., Chaventre, A., Elion, J., et al. (2001) Commensal Escherichia coli isolates are phylogenetically distributed among geographically distinct human populations. Microbiology 147: 16711676.

Escobar-Páramo, P., Clermont, O., Blanc-Potard, A., Bui, H., Le Bouguenec, C., and Denamur, E. (2004a) A specific genetic background is required for acquisition and expression of virulence factors in Escherichia coli. Mol Biol Evol 21: 1085-1094.

Escobar-Páramo, P., Grenet, K., Le Menach, A., Rode, L., Salgado, E., Amorin, C., et al. (2004b) Large-scale population structure of human commensal Escherichia coli isolates. Appl Environ Microbiol 70: 5698-5700.

Escobar-Páramo, P., Sabbagh, A., Darlu, P., Pradillon, O., Vaury, C., Denamur, E., and Lecointre, G. (2004c) Decreasing the effects of horizontal gene transfer on bacterial phylogeny: the Escherichia coli case study. Mol Phylogenet Evol 30: 243-250.

Escobar-Páramo, P., Le Menac'h, A., Le Gall, T., Amorin, C., Gouriou, S., Picard, B., et al. (2006) Identification of forces shaping the commensal Escherichia coli genetic structure by comparing animal and human isolates. Environ Microbiol 8: 1975-1984.

Gerdes, S.Y., Scholle, M.D., Campbell, J.W., Balazsi, G., Ravasz, E., Daugherty, M.D., et al. (2003) Experimental determination and system level analysis of essential genes in Escherichia coli MG1655. J Bacteriol 185: 56735684.

Gordon, D.M. (2001) Geographical structure and host specificity in bacteria and the implications for tracing the source of coliform contamination. Microbiology 147: 1079-1085.

Gordon, D.M., and Cowling, A. (2003) The distribution and genetic structure of Escherichia coli in Australian verte- 
brates: host and geographic effects. Microbiology 149: 3575-3586.

Gordon, D.M., and O'Brien, C.L. (2006) Bacteriocin diversity and the frequency of multiple bacteriocin production in Escherichia coli. Microbiology 152: 3239-3244.

Gordon, D.M., Stern, S.E., and Collignon, P.J. (2005) Influence of the age and sex of human hosts on the distribution of Escherichia coli ECOR groups and virulence traits. Microbiology 151: 15-23.

Hejnova, J., Dobrindt, U., Nemcova, R., Rusniok, C., Bomba, A., Frangeul, L., et al. (2005) Characterization of the flexible genome complement of the commensal Escherichia coli strain A0, 34/86 (O83: K24: H31). Microbiology 151: 385-398.

Herzer, P.J., Inouye, S., Inouye, M., and Whittam, T.S. (1990) Phylogenetic distribution of branched RNA-linked multicopy single-stranded DNA among natural isolates of Escherichia coli. J Bacteriol 172: 6175-6181.

Johnson, J.R., and Stell, A.L. (2000) Extended virulence genotypes of Escherichia coli strains from patients with urosepsis in relation to phylogeny and host compromise. $J$ Infect Dis 181: 261-272.

Johnson, J.R., Clermont, O., Menard, M., Kuskowski, M.A., Picard, B., and Denamur, E. (2006) Experimental mouse lethality of Escherichia coli isolates, in relation to accessory traits, phylogenetic group, and ecological source. $J$ Infect Dis 194: 1141-1150.

Le Gall, T.O., Clermont, S., Gouriou, B., Picard, X., Nassif, E., Denamur and Tenaillon, O. (2007) Extraintestinal virulence is a coincidental by-product of commensalisms in B2 phylogenetic group Escherichia coli strains. Mol Biol Evol 24: 2373-2384. August 19. Epub ahead of print.

Leyton, D.L., Adams, L.M., Kelly, M., Sloan, J., Tauschek, M.,
Robins-Browne, R.M., and Hartland, E.L. (2007) Contribution of a novel gene rpeA encoding a putative autotransporter adhesin to intestinal colonization by rabbit enteropathogenic Escherichia coli. Infect Immun 75: 46644669.

Ochman, H., and Selander, R.K. (1984) Standard reference strains of Escherichia coli from natural populations. J Bacteriol 157: 690-693.

Picard, B., Garcia, J., Gouriou, S., Duriez, P., Brahimi, N., Bingen, E., et al. (1999) The link between phylogeny and virulence in Escherichia coli extraintestinal infection. Infect Immun 67: 546-553.

Power, M.L., Littlefield-Wyer, J., Gordon, D.M., Veal, D.A., and Slade, M.B. (2005) Phenotypic and genotypic characterisation of encapsulated Escherichia coli isolated from blooms in two Australian lakes. Environ Microbiol 7: 631640.

Scott, T.M., Rose, J.B., Jenkins, T.M., Farrah, S.R., and Lukasik, J. (2002) Microbial source tracking: current methodology and future directions. Appl Environ Microbiol 68: 5796-5803.

Souza, V., Rocha, M., Valera, A., and Eguiarte, L.E. (1999) Genetic structure of natural populations of Escherichia coli in wild hosts on different continents. Appl Environ Microbiol 65: 3373-3385.

Sun, J., Gunzer, F., Westendorf, A.M., Buer, J., Scharfe, M., Jarek, M., et al. (2005) Genomic peculiarity of coding sequences and metabolic potential of probiotic Escherichia coli strain Nissle 1917 inferred from raw genome data. J Biotechnol 117: 147-161.

Wirth, T., Falush, D., Lan, R., Colles, F., Mensa, P., Wieler, L.H., et al. (2006) Sex and virulence in Escherichia coli: an evolutionary perspective. Mol Microbiol 60: 1136-1151. 